\title{
Application of seismic isolation in the retrofit of historical buildings
}

\author{
P. Clemente ${ }^{1} \&$ A. De Stefano ${ }^{2}$ \\ ${ }^{1}$ ENEA, Rome, Italy \\ ${ }^{2}$ Politecnico di Torino, Turin, Italy
}

\begin{abstract}
In this paper the studies carried out for the retrofit of Margherita Palace, seriously damaged by L'Aquila earthquake, are shown. The buildings and the tower have been first analyzed by means of ambient vibration tests, in order to find out the dynamic characteristics of the structure even in a damaged situation. On the basis of the results obtained and of the microzoning analysis carried out after the seismic event, the base isolation has been proposed as the most suitable solution for the seismic retrofit of the structure, because allows to obtain a good structural result without compromising the architectural characteristic of the superstructure. A new isolation system is then proposed for the seismic retrofit of historical buildings, based on the realization of an isolated platform under the building foundation without any intervention on the building. The system can be used for single buildings but also for complex structure, typical of Italian historical centres.
\end{abstract}

Keywords: seismic isolation, historical buildings, cultural heritage.

\section{Introduction}

The traditional techniques, based on the increasing of strength and ductility, are not suitable for the seismic rehabilitation of cultural heritage buildings for the following reasons:

- they are often not reversible,

- they make use of materials different and incompatible with the original ones,

- they change the original structural conception. 
The last aspect is sometimes compulsory, because the building was design without accounting for the seismic actions, being vulnerable even to moderate events. Besides, historical buildings often present weak points, such as an irregular form both in plan and in elevation, the lack of vertical joints and transversal braces, in-plane flexibility of floor slabs and shallow foundations.

Furthermore, under earthquakes of high intensity, traditional structures can just guarantee against the collapse, but cannot avoid heavy damages both to structural and non-structural elements. As a result, for cultural heritage buildings, a suitable equilibrium between the two opposite requirements is usually accepted, i.e. a partial seismic improvement is obtained preserving their original monumental characteristics, identity and historical value.

It is worth noting that, due to the historical importance and to the daily presence of tourists, the seismic rehabilitation of historical buildings is quite delicate, aiming at the protection of both human life and cultural heritage.

Base isolation could be a suitable solution for the rehabilitation of historical structures. It aims to reduce seismic actions, thus avoiding significant damages to the structure and its contents even under strong earthquakes, and presents very low interference with the structure itself.

In this paper the seismic isolation of an earthquake damaged building in L'Aquila is proposed. First of all an experimental campaign has been carried out in order to find out the dynamic characteristics of the structure. Then a conventional improvement intervention has been defined, which allows the structure to be able to support minimum horizontal actions. On the basis of the effective earthquake resistance of the restored structure, several solutions for the base isolation system have been designed.

\section{Margherita Palace and the Civic Tower}

The erection of the City Hall in L'Aquila started in 1294 and a first important restoration was completed in 1541 (Fig. 1). Important works were done since 1573, when the building became the house of Margherita d'Austria, and most of the original characteristics were lost. At the beginning of the $20^{\text {th }}$ century another
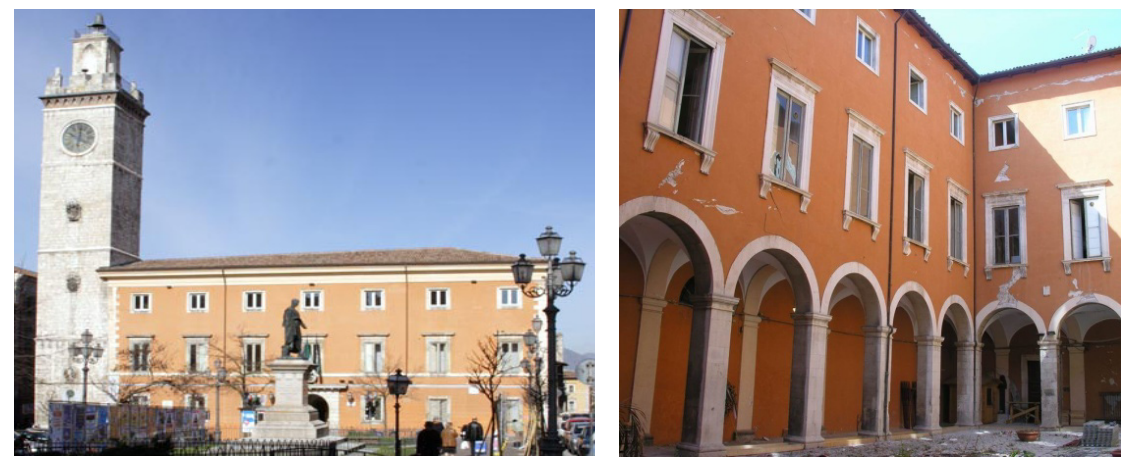

Figure 1: $\quad$ Margherita Palace: main facade and courtyard. 
restoration intervention was done to realize a concrete ring beam under the roofing. The palace has two levels and rectangular plan of about $40 * 60 \mathrm{~m}$, with an internal court. The vertical structure is made of stone and brick masonry with good mortar. The horizontal structures are made of masonry vaults, some of them with chains, and steel decks. Underground levels could be present under some parts of the building.

The Civic Tower, built from 1254 and 1374 at the N-E corner, was seriously damaged by the 1703 earthquake and rebuilt with lower height. In 1937 the tower was consolidated by inserting iron T-beams at the floors. It has an almost square cross-section of about $6.30 \mathrm{~m}$. The thickness of the walls is equal to $2.0 \mathrm{~m}$ at the basement.

The palace suffered heavy damages during L'Aquila earthquake of April $6^{\text {th }}$, 2009. Several cracks are apparent and local collapse mechanisms have been activated. In more details the seismic events caused the disconnection between the orthogonal walls, the out of plane collapse of some masonry walls, the formation of large cracks, collapse of some floors and important damages to the stairs.

\section{Dynamic characterization}

Fifteen seismometers (velocimeter sensors) have been used, deployed in two configurations, the first one relative to the building and the second to the tower.

In the first one, the sensors have been deployed in the building in order to point put the global resonance frequencies and any mode of each part (Fig. 2). In the second configuration sensors have been deployed on different levels of in the tower.

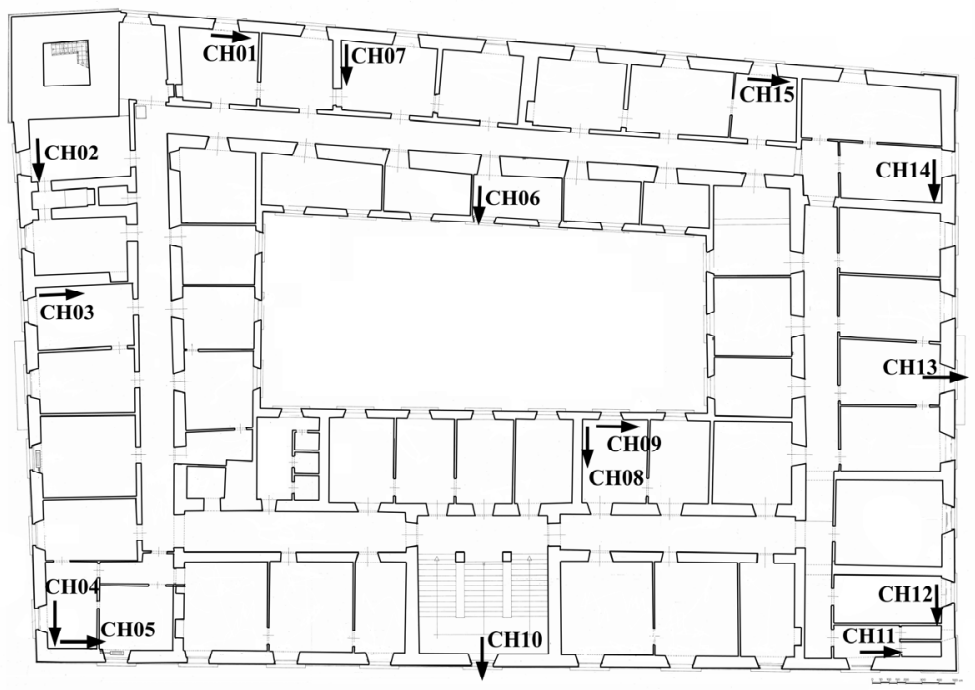

Figure 2: $\quad$ Second floor: sensor deployment. 
For each configuration three tests of about $300 s$ have been carried out, with a sample rate of $200 \mathrm{point} / \mathrm{s}$ using ambient noise only as source of vibrations. With this low level of excitation the building showed a quasi-linear behaviour and a spectral analysis could be performed. The data has been analyzed in the frequency domain by plotting the power spectral density (PSD) of each record and the cross spectral densities (CSD), in terms of amplitude and phase factor, of selected couples of records with the corresponding coherence functions. As well known, peaks in PSD could be associated to structural frequencies while the same peaks in CSD, with value of phase factor equal to $0^{\circ}$ or $180^{\circ}$, and value of coherence function close to unity, confirm this statement and give some indications on the modal shape associated to each structural frequency.
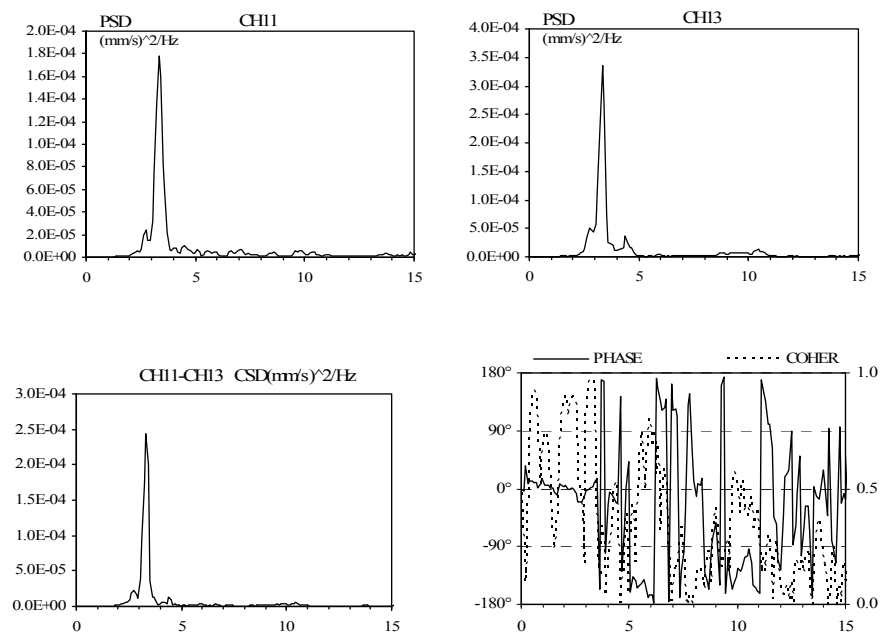

Figure 3: $\quad$ PSD and CSD of longitudinal sensors.

In Fig. 3 the PSDs and the CDS relative to sensors $\mathrm{CH} 11$ and $\mathrm{CH} 13$ in the first configuration are plotted. The experimental analysis pointed out the resonance frequencies of the building listed in Tab. 1.

Table 1: $\quad$ Resonance frequencies of the building.

\begin{tabular}{|c|c|}
\hline Freq. $(\mathrm{Hz})$ & Description \\
\hline 2.24 & Transversal \\
\hline 2.74 & Transversal + Tors. \\
\hline 3.32 & Longitudinal \\
\hline 3.71 & Torsional \\
\hline
\end{tabular}



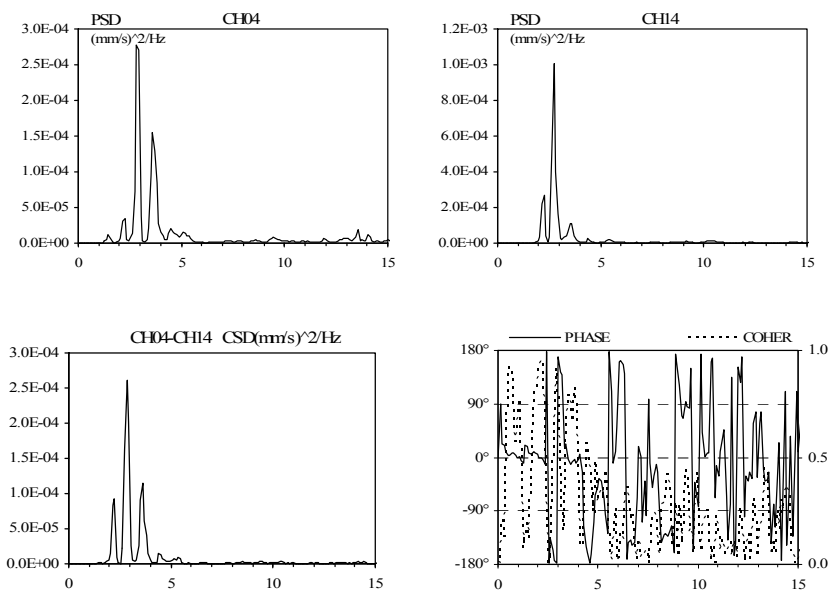

Figure 4: $\quad$ PSD and CSD of transversal sensors.

In Fig. 4 the PSDs and the CDS relative to the second configuration are shown. The experimental analysis pointed out the resonance frequencies of the tower listed in Tab. 2.

Table 2: $\quad$ Resonance frequencies of the tower.

\begin{tabular}{|c|c|}
\hline Freq. $(\mathrm{Hz})$ & Description \\
\hline 1.46 & Transversal \\
\hline 1.46 & Longitudinal \\
\hline 2.83 & Transversal \\
\hline 3.22 & Longitudinal \\
\hline 4.20 & Longitudinal \\
\hline 4.98 & Transversal \\
\hline 5.66 & Longitudinal \\
\hline
\end{tabular}

\section{The base isolation system}

The obtained values must be taken into account in the design of the isolation system, in order to obtain a suitable decoupling between the motion of the building and the motion of the soil. As well known, in fact, the frequency of the isolated building should be lower enough with reference to the frequency of the superstructure, usually not higher than one third of it.

Besides, it is important reminding that the seismic microzoning, carried out by the Italian National Civil Protection Department, pointed out the presence of seismic amplifications in the range $0.4 \div 0.6 \mathrm{~Hz}$. So the main resonance frequencies of the isolated system should be lower than the minimum value of this range. 
Finally, the isolation system should be designed in order to reduce the seismic action in the structure to the value that the restored building will be able to support in the elastic range.

In more details, the frequency of the isolated building, or its first period of vibration, is chosen as the one corresponding, in the elastic spectrum relative to the life limit state (SLV), to the value of acceleration which can affects the superstructure.

From a preliminary analysis a value of $0.10 \mathrm{~g}$ has been fixed as the maximum spectral acceleration in the superstructure and the corresponding period in the SLV spectrum has been assumed as the period of the isolated building. Then the corresponding acceleration and displacements values in the collapse limit state (SLC) spectrum has been evaluated (Fig. 5). Taking into account also what said about the local amplification, the isolation period has been fixed equal to $3.0 \mathrm{~s}$. For this value of the period, $\mathrm{tt}$ is $S_{e}=0.07 \mathrm{~g}$ and $S_{D e}=0.30 \mathrm{~m}$.

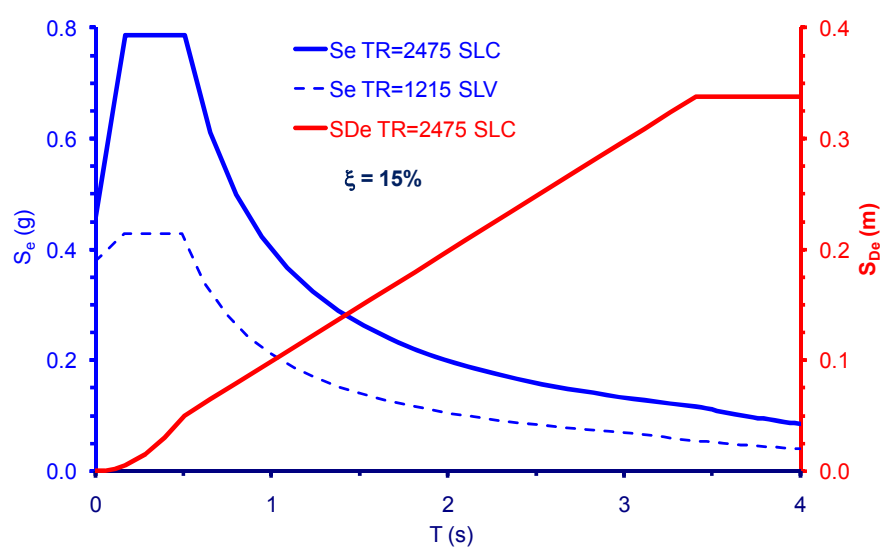

Figure 5: $\quad$ SLV and SLC spectra.

Different solutions have been considered for the seismic retrofit of the structure. In the following only two of them will be shown, the most traditional and the most innovative one, respectively.

\subsection{Insertion of a base isolation system in the existing structure}

In the first solution the isolation system has been inserted just below the ground level of the building. A gap between the masonry walls and the foundations can be created by cutting the masonry walls at their basement, as shown in figs. 6 and 7 and realizing two concrete beams under and above the isolators.

The construction phases are the following:

- realization of windows in the masonry walls at the isolation device positions. The window should be high enough to contain a lower and a upper beams and the isolator (Fig. 6); 
- construction of the portion of the lower concrete beam, with the overlap steel bar between the beam portion of the considered window and the next ones;

- installation of the isolation device;

- realization of the portion of the upper concrete beam, with the overlap steel bar between the adjacent windows;

- cutting of masonry between the windows;

- connection of the lower concrete beam portions and of the upper ones (Fig. 7).

This solution requires traditional works and is not very expensive, but presents the disadvantages of requiring the modification of the foundations and of being not reversible. So it is not always applicable in practice for historical constructions.

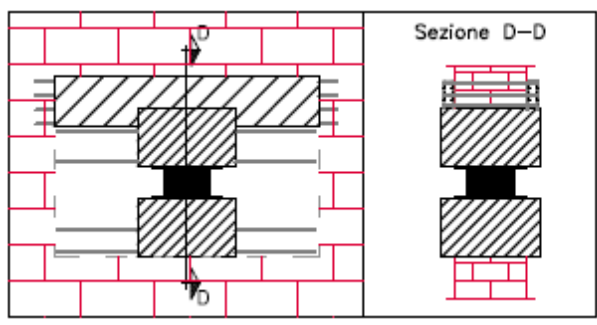

Figure 6: Window in the wall.

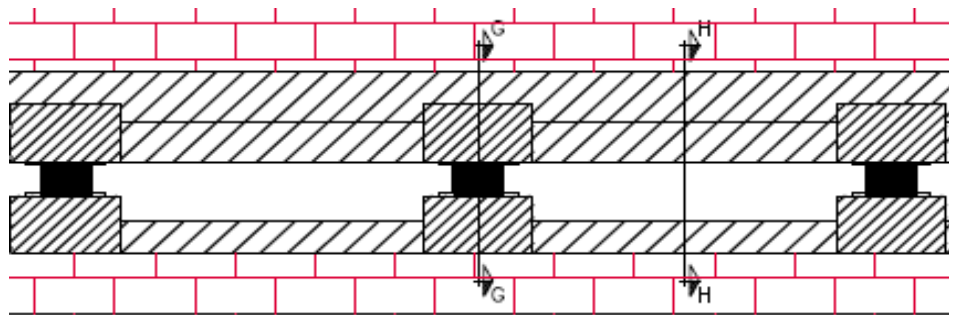

Figure 7: $\quad$ Isolation devices, lower and upper beams.

\subsection{A new solution}

The new solution proposed consists in the realization of an isolated platform under the foundations of the building, without touching the building itself (Fig. 8). A discontinuity between the foundations and the soil is created by means of the insertion of horizontal pipes and the positioning of isolation devices at the horizontal diametric plane. Then the building is separated from the surrounding soil in order to allow the horizontal displacements required by the isolation system. So the structure is seismically isolated but not interested by interventions 
that could modify its architectural characteristics, which is very important for historical buildings.

Even underground level are not modified but can be part of the seismically protected building. In more details the construction phases are the following (Fig. 9):

- a trench is first excavated of at one side of the building and pipes are inserted by means of auger boring or micro-tunnelling technique; the diameter of pipes should be $\geq 2 \mathrm{~m}$, in order to allow the inspection of the isolation system;

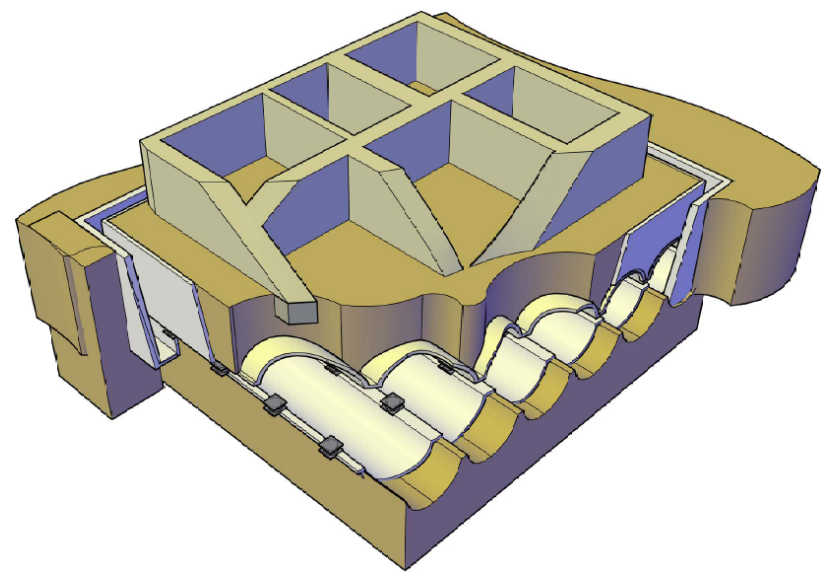

Figure 8: The new isolation system.
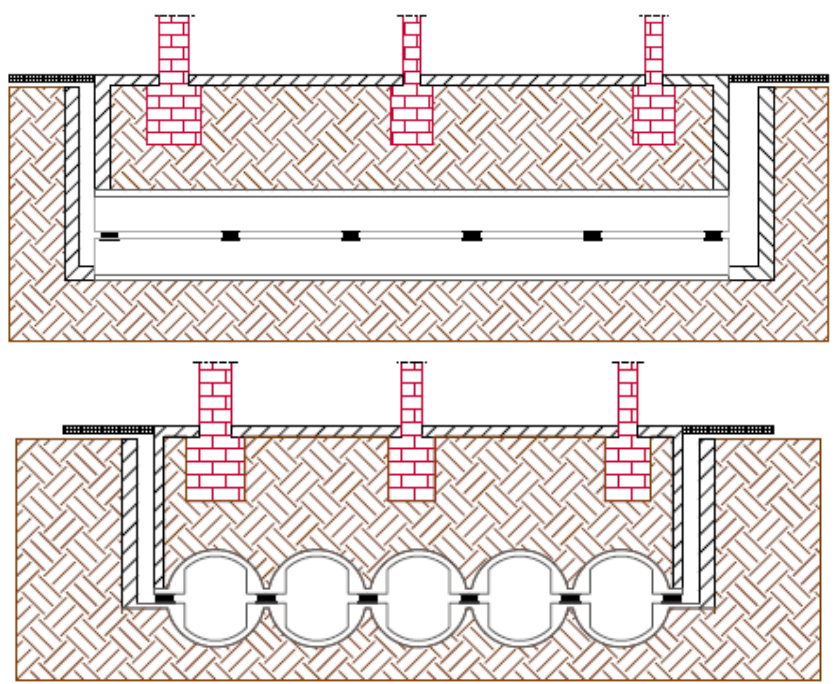

Figure 9: Longitudinal and transversal cross-sections of the new isolation system. 
The pieces of pipe should have a particular shape and are composed by two portions, the lower and the upper sectors, respectively, which are connected by means of removable elements.

- the connection elements placed in correspondence of the isolation devices are removed and each pipe is joined with the two adjacent ones, for example by means of a reinforced concrete elements;

- the isolation devices are positioned and the upper adjacent sectors are connected in correspondence of the isolators;

- successively also the other connection elements are removed, so the lower and upper sectors are definitely separated;

- finally vertical walls are built along the four sides of the building and a rigid connection, a concrete slab or other, is realized between the building and the isolation system.

The system allows also the realization of a tunnel for pedestrian or vehicles. The size of the pipes must guarantee the accessibility and the possibility to substitute the devices.

It is worth reminding that the solution presents the advantages that the building and its architectural aspect are not changed and so are the underground levels; this is a very important requirement for historical and monumental structures.

\section{Problems to consider: vibrations and settlements}

During the micro-tunnelling operation two problems can arise: the soil settlement and the vibration induced at the surface level.

The literature related to the vibrations induced by micro-tunnelling is not very ample and often not strictly pertinent. Some suggestions can come from analogue experiences supplied by large tunnelling works or from vertical boreholes. These experiences suggest that minor threats should be expected from induced vibrations, but theoretical and experimental deeper studies are needed.

More serious problems can arise by settlements [1-3]. To reason concretely in a quantitative manner we will refer to a specific case study, which is Margherita Palace, the City Hall in L'Aquila, where, thanks to a previous experimental dynamic soil characterization at a near site, it is possible to model the mechanical properties of the ground with accuracy. A FE 2-D model has been set up and then exploited in a Diana 2 environment. The model is necessarily at list a 2-D model, because it shall be useful to analyze a perturbation of limited width. The perturbation is due to the foundation of a building and to the micro-tunnels under it. The vertical edges of the model are kept far enough from the perturbed zone, to reduce their influence as much as possible. The nodes belonging to those edges are restrained by means of spring and dampers able to cut-off the wave reflection.

The dynamic characterization shows that stiffness and strength increase with depth. In the model the soil is described as a layered continuum indefinitely extended, supported by the bedrock at $17 \mathrm{~m}$ depth. Each layer is $1.0 \mathrm{~m}$ thick and 
its elastic dynamic tangential modulus $G_{0}$ is coherent with the measured wave propagation velocities:

$$
G_{0}=\rho \cdot v_{s}^{2}
$$

The Young dynamic and static modulus is given, respectively, by:

$$
E_{d y n}=G_{0} \cdot 2 \cdot(1+v) ; \quad E_{\text {static }}=E_{d y n} / 3 ; \quad(v=0.3)
$$

To realize the mesh of the FE model, eight node quadrangular elements have been used, paying attention to keep the aspect ratio near to one and regular as much as possible (Fig. 10). The plane deformation condition is imposed and the boundary nodes respect the following restraining conditions: the vertical displacement is inhibited for the nodes belonging to the lower horizontal edge; the horizontal displacement is inhibited for the nodes belonging to both the lateral vertical edges. A mass density of $2090 \mathrm{~kg} / \mathrm{m}^{3}$ has been assumed.

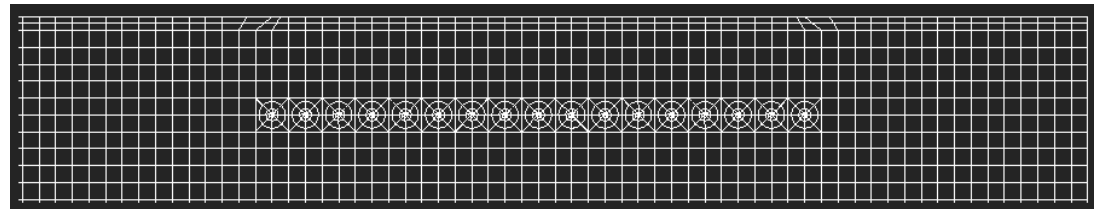

Figure 10: The sub-soil 2-D FE model.

If local perturbations due to building foundation and micro-tunnels are absent, the response of the model to horizontal base shaking or vertical uniform static pressure from the top level are comparable to the response, in similar conditions, of a multi-layered mono-dimensional model, except in proximity of the lateral vertical edges. The successful validation of the model has been based on this criterion.

On the validated model, the building imposes a load of $3000 \mathrm{kN} / \mathrm{m}$ uniformly distributed along its base width. That load induces a local settlement.

Then micro-tunnels, later named simply "pipes", are included in the model, following two different alternative strategies:

1. First strategy: one central pipe, then the two most external ones and all the other filling the layer from the external pipes to de centre.

2. Second strategy: one central pipe, then the two most external ones; other pipes are then inserted in intermediate positions, regularly spaced, filling gradually the layer.

The advances following the two strategies are shown in Figure 11a and b, respectively, where the settlement is also represented.

The boreholes induce an additional settlement that is the one that we are interested to estimate. The problem being non-linear, the settlement due to insertion of pipes, originated by a stress release process, is computed as difference between the settlement due to the weight of the building and the insertion of the pipes and the settlement due to the weight of the building alone. 


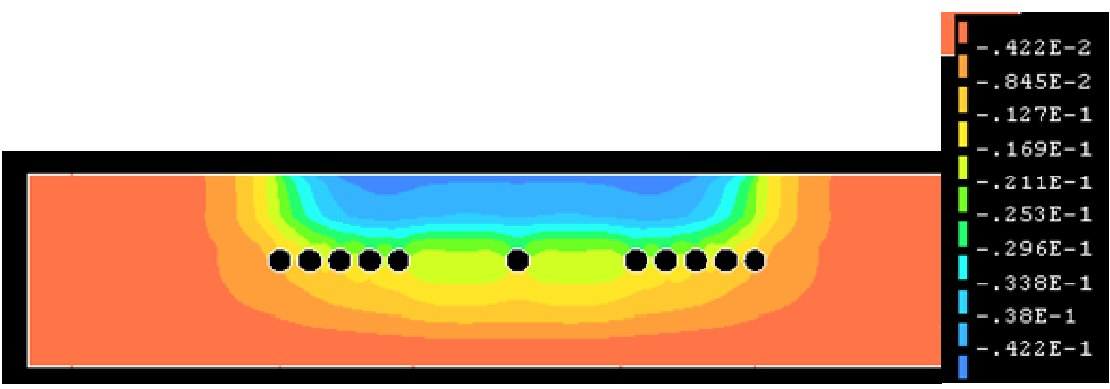

a) strategy 1

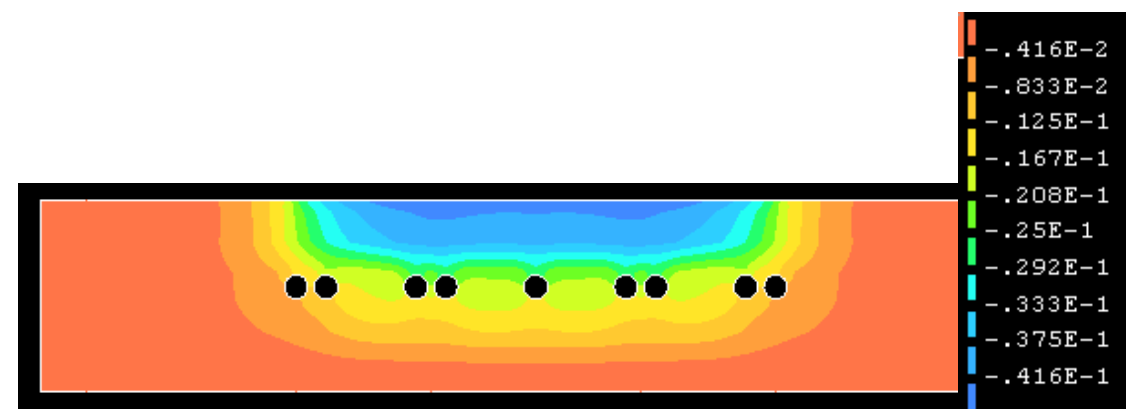

b) strategy 2

Figure 11: Filling the layer of pipes.

The stress release during micro-tunnelling is a three-dimensional mechanism that shall be described by a plane-strain two-dimensional model, as previously stated. It is possible to reach that goal through a conventional hole-boundary force reduction approach known as " $\beta$-value method" or Convergenceconfinement method proposed by Panet and Guenot (1982) using the stressrelease factor $\lambda$, varying inside the $0-1$ range [1].

Analyses are then based on the decrement of a fictitious internal pressure at the boundary of the holes in agreement with the $\beta$-value method. To apply that method inside the FE model simply supporting elastic restrains are distributed along the hole boundaries, with operating direction orthogonal to them. By modifying the stiffness of the elastic supports it is possible to simulate the stress release. A null stiffness of the elastic support gives $\lambda=1$.

For the actual case study we assume $\lambda=0.4$ and $H / D=3.5$, where $H$ is the depth of the pipe axis and $\mathrm{D}$ is the diameter of the pipe.

The strategy 1 is easier to apply, but the effect on soil adaptation is not so smooth and regular. The final computed value of the settlement is about $6.8 \mathrm{~mm}$. The strategy 2 leads to more regular advancement and allows a slight settlement reduction to about $5.6 \mathrm{~mm}$. Increasing the stress release factor $\lambda$ to 0.6 the settlement values increase nearly with a multiplier 1.2. A larger $H / D$ ratio reduces the problem but increases the cost of the trenches. Technologies to contrast the settlements exist and are consolidated but, of course, they push the cost up. 


\section{Conclusions}

A new isolation system has been proposed for the seismic retrofit of historical buildings, based on the realization of an isolated platform under the building foundation without any intervention on the building. The system can be used for single buildings but also for complex structure, typical of Italian historical centers. In fact, the ability of the proposed system to work "under all" can make it useful also for every situation in which separate buildings or components are connected by complex lifelines, pipes and other links. Such condition is usual, for instance, in chemical and power production plants, including the nuclearones.

\section{Acknowledgements}

The authors thank G. Barla for his important support and contribution and the student Stefano Renna (Politecnico di Torino) who allowed them to use the results of his studies on the soil adaptation and settlements, extracted from his Master Thesis final work.

\section{References}

[1] Barla, G. B., Viggiani, G., Le opere in sotterraneo. Proc. of the XXI Italian National Conference of Geotechnics (in Italian), Vol. II, 2002.

[2] Miliziano, S., Soccodato, F.M., Burghignoli, A., Evaluation of damage in masonry buildings due to tunnelling in clayey soils. Proc. 3rd Int. Symp. on Geotechnical Aspects of Underground Construction in Soft Ground, Toulouse, France, pp. 335-340. 2002.

[3] Barla, M., Camusso, M., A method to design microtunnelling installations in the Torino randomly cemented alluvial soil. Tunnelling and Underground Space Technology, ISSN 0886-7798, in print, 2011. 